\title{
Weshalb die EU nicht zur sozialen Marktwirtschaft werden kann
}

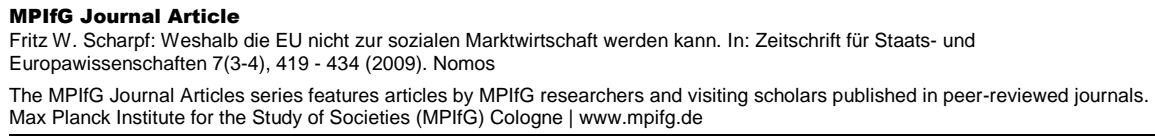

von Fritz W. Scharpf

Die europäische Integration ist nicht nur durch politische Initiativen, sondern auch durch das Richterrecht vorangetrieben worden, das - anders als die europäische Gesetzgebung - nicht durch hohe Konsenshürden behindert wird. Für sich genommen kann die Rechtsprechung jedoch nur die nationalen Rechtsordnungen deregulieren und liberalisieren. Sie traf deshalb in besonderem Maße die Mitgliedstaaten, deren sozioökonomische Ordnung den Werten einer "sozialen Marktwirtschaft" entsprach. Zwar postuliert nun der Lissabon-Vertrag die europäische „,soziale Marktwirtschaft“ als Ziel der Union. Die dafür notwendige europäische Gesetzgebung aber wird auch nach dem Übergang zu qualifizierten Mehrheitsvoten im Rat durch das Veto der Mitgliedstaaten mit einer ,, liberalen Marktwirtschaft" blockiert. Im Ergebnis wird die Wirtschaftsordnung der Union deshalb dem liberalen Modell entsprechen.

European integration has been the joint product of political initiatives and judicial action which, in contrast to European legislation, was never impeded by high consensus requirements. By itself, however, adjudication was only able to deregulate and liberalise national institutions and rules that interfered with European economic liberties and other Treaty-based subjective rights. Its impact was particularly damaging in member states whose socio-economic orders reflected the values of a "social market economy". Now the Lisbon Treaty has postulated the European "social market economy" as a goal of the Union. But the European legislation that would be required to promote this goal still depends on qualified majorities in the Council and will be easily blocked by the veto of member states preferring a "liberal market economy". Hence the socio-economic regime of the European economy is bound to conform to the "liberal" model.

Im Lissabon-Vertrag wurde, nicht zuletzt auf deutsches Betreiben, die Verwirklichung einer ,,in hohem Maße wettbewerbsfähigen sozialen Marktwirtschaft, die auf Vollbeschäftigung und sozialen Fortschritt abzielt“ unter die ,Ziele der Union" aufgenommen (Art. 3 Abs. 3 EUV). Im Folgenden soll gezeigt werden, welche Hindernisse der Erreichbarkeit dieses Zieles entgegenstehen und dass diese in erster Linie dem europäischen Richterrecht zuzuschreiben sind, dessen wichtiger Beitrag zur europäischen Integration zugleich eine gegen die Institutionen der sozialen Marktwirtschaft gerichtete Dynamik erzeugt hat. 
Die europäische Integration schuldet ihren Erfolg gewiss der Weitsicht und Kraft großer Politiker, Schuman, Monnet, de Gasperi, Spaak, Adenauer und anderen, die aus den Ruinen des Zweiten Weltkriegs das gemeinsame europäische Haus bauen wollten - von der Montanunion über die gescheiterte Europäische Verteidigungsgemeinschaft bis zu den Römischen Verträgen und der Gründung der Europäischen Wirtschaftsgemeinschaft (EWG). Und selbstverständlich erforderten auch die folgenden Schritte zur Erweiterung und Vertiefung der Integration immer wieder das Engagement der führenden Politiker und den riskanten Einsatz ihres politischen Kapitals. Anders hätten weder die aufeinander folgenden Schübe der Erweiterung von einer Gemeinschaft der sechs zur Union der 27 erreicht werden können, noch die Vertiefung von der Zollunion zum Binnenmarkt, zur Wirtschafts- und Währungsunion und schließlich zum Verfassungsvertrag von Lissabon. Die Integration ist also das aller wohlfeilen Skepsis widersprechende Werk von demokratisch verantwortlichen Politikern in den europäischen Ländern, die von der Adenauer-Generation bis zur Generation ihrer Urenkel und über unzählige Regierungswechsel hinweg an der Vision eines geeinten Europas festhielten und die trotz aller Hindernisse die jeweils sich bietenden historischen Chancen zur Vertiefung und Erweiterung nutzten. ${ }^{1}$ Aber dies ist nicht die ganze Geschichte.

\section{Die Rolle des europäischen Richterrechts}

Auch wenn die großen „konstitutionellen“ Entscheidungen der europäischen Integration immer Sache der nationalen Regierungen blieben, so sind doch die Richtung und die realen Wirkungen der tatsächlich verfolgten europäischen Politik in weit höherem Maße durch die Europäische Kommission und den Europäischen Gerichtshof (EuGH) bestimmt worden. Dabei kam dem EuGH eine Schlüsselrolle zu, die zwar von der Öffentlichkeit und auch von der Politikwissenschaft lange übersehen wurde, aber von den Spezialisten des Europarechts früh erkannt und als ,Integration through Law “ gefeiert wurde. ${ }^{2}$

Darin lag keine Übertreibung. In der Tat war der politische Integrationsprozess in den siebziger Jahren ins Stocken gekommen. Unter französischem Druck hatte der „Luxemburger Kompromiss“ die Einstimmigkeit im Ministerrat festgeschrieben; mit der ersten Erweiterung um Großbritannien, Irland und Dänemark 
hatten sich institutionellen, politischen, kulturellen und ökonomischen Gegensätze zwischen den Mitgliedstaaten enorm vergrößert; und überdies hatten diese im Gefolge der ersten Ölpreiskrise mit rapide ansteigenden Inflations- und Arbeitslosigkeitsraten zu kämpfen. Statt um die Beseitigung von Handelshindernissen ging es den Mitgliedstaaten damals um den Schutz der heimischen Wirtschaft, und der vorgesehene Übergang von der Zollunion zum gemeinsamen Binnenmarkt schien in weite Ferne gerückt.

In dieser Situation, als der Ministerrat blockiert war und eine Überwindung der „Eurosklerose“ durch politische Initiativen unwahrscheinlich erschien, öffnete die Rechtsprechung einen Weg, auf dem die Integration trotzdem vorangetrieben werden konnte. Die rechtlichen Voraussetzungen dafür hatte das Gericht schon in den frühen 1960er Jahren geschaffen, als es gegen den Widerstand einiger Regierungen die Direktwirkung ${ }^{3}$ des Europarechts und seinen Vorrang ${ }^{4}$ vor allem nationalen Recht postuliert hatte - und dafür auch die Folgebereitschaft der nationalen Gerichte gefunden hatten. ${ }^{5}$ Ihre volle Durchschlagskraft gewannen diese Prinzipien jedoch dadurch, dass das Gericht zugleich die vertragliche Verpflichtung der Mitgliedstaaten zur Beseitigung von Zöllen und zur Herstellung des freien Verkehrs von Waren, Dienstleistungen, Kapital und Personen in subjektive Individualrechte umdeutete. Diese „wirtschaftlichen Grundfreiheiten“ konnten also von Einzelpersonen und Unternehmen in normalen Rechtsstreitigkeiten gegen nationales Recht in Anspruch genommen werden, und die befassten Gerichte waren gehalten, bei Zweifeln über deren Auslegung die verbindliche „Vorabentscheidung“ des EuGH einzuholen (Art. 234 EGV).

Die Anwendung beschränkte sich freilich zunächst auf Zölle und andere Regeln, die eine unmittelbare Diskriminierung ausländischer Anbieter bezweckten. Dagegen gingen die Regierungen und die Kommission davon aus, dass nichtdiskriminierende nationale Regeln, die aber wegen ihrer Unterschiedlichkeit als nichttarifärer Handelshindernisse wirkten, nur durch Harmonisierung - also durch einstimmig zu verabschiedende Richtlinien - zu überwinden waren. ${ }^{6}$ Aber als der Rat in den 1970er Jahren kaum noch in der Lage war, sich auf solche Richtlinien zu einigen, da lagen die juristischen Instrumente zur „Ersatzvornahme“

3 EuGH, Urt. v. 05.02. 1963, Rs. 26/62 (Van Gend \& Loos), Slg. 1963, 3.

4 EuGH, Urt. v. 15.07. 1964, Rs. 6/64 (Costa v. Enel), Slg. 1964, 1141.

5 Alter, K.J.: Establishing the Supremacy of European Law, Oxford, 2001.

6 Haltern, U.: Europarecht, 2. Aufl., Tübingen, 2007. 
bereit, und mit der Entscheidung im berühmten Cassis-Fall ${ }^{7}$ trat das Richterrecht an die Stelle des politischen Gesetzgebers: Nationale Regeln, die den freien Warenverkehr behinderten, waren nicht länger anwendbar. An deren Stelle trat das Pflicht der Mitgliedstaaten zur „wechselseitigen Anerkennung“ der Regeln des jeweiligen Herkunftslandes.

Paradoxerweise stieß dieser revolutionäre Akt der richterlichen Rechtssetzung bei den Regierungen kaum auf Widerstand, sondern wirkte stattdessen - von der Kommission strategisch genutzt - als massiver Impuls zur Erneuerung der politischen Integration, weil das Urteil die Verhandlungssituation im Rat radikal verändert hatte. Während zuvor das nationale Recht weiter galt, wenn eine Harmonisierungsrichtlinie scheitere, war nun dessen Bestand prekär geworden. Angesichts der extrem weiten Definition der „Grundfreiheiten“ im vorangegangenen Dassonville-Urteil ${ }^{8}$ konnten nun jede nationale Regelung als ,direkte oder indirekte, tatsächliche oder potentielle" Behinderung des freien Verkehrs angegriffen werden, und es lag allein im Ermessen des Gerichts, ob die behauptete Behinderung ausnahmsweise durch die Berufung auf ,zwingende Erfordernisse des Allgemeininteresses ${ }^{\star 9}$ gerechtfertigt werden konnte. ${ }^{10}$ Angesichts dieser Konstellation konnten die Regierungen durch die Blockade von Harmonisierungs-Richtlinien nichts mehr gewinnen, und angesichts drohender Rechtsunsicherheit musste die konsensual geregelte Harmonisierung als das kleinere Übel erscheinen. ${ }^{11}$ Das Ergebnis war die Einheitliche Europäische Akte von 1986 mit dem Übergang zur Abstimmung mit qualifizierter Mehrheit im Rat bei Maßnahmen zur Vollendung des Binnenmarktes (Art. 95 EGV) - und in der Folge eine wahre Flut von harmonisierenden Richtlinien.

7 EuGH, Urt. v. 20.02. 1979, Rs. 120/78 (Rewe v. Bundesmonopolverwaltung für Branntwein), Slg. 1979, 649.

8 EuGH, Urt. v. 11.07.1974, Rs. 8/74 (Dassonville), Slg. 1974, 837.

9 Die Standard-Formel der potenziellen Rechtfertigungsgründe findet sich in der Gebhard-Entscheidung: EuGH, Urt. v. 30.11.1995, Rs. 55/94 (Gebhard), Slg. 1995, I-4165. Demnach müssen „nationale Maßnahmen, die die Ausübung der durch den Vertrag garantierten grundlegenden Freiheiten behindern oder weniger attraktiv machen können, vier Voraussetzungen erfüllen: Sie müssen in nichtdiskriminierender Weise angewandt werden, sie müssen aus zwingenden Gründen des Allgemeininteresses gerechtfertigt sein, sie müssen geeignet sein, die Verwirklichung des mit ihnen verfolgten Zieles zu gewährleisten, und sie dürfen nicht über das hinausgehen, was zur Erreichung dieses Zieles erforderlich ist.“

10 Haltern, U., a. a. O.

11 Alter, K.J./Meunier-Aitsahalia, S.: Judicial Politics in the European Community: European Integration and the Pathbreaking Cassis de Dijon Decision, in: Comparative Political Studies, 26/4 (1994), 535561; Schmidt, S. K.: Beyond Compliance: The Europeanization of Member States through Negative Integration and Legal Uncertainty, in: Journal of Comparative Policy Analysis, 10/3 (2008), 299-308. 
Aber die „Integration durch (Richter-)Recht“ hatte damit keineswegs ihr Ende gefunden. Zunächst stützte sie sich zwar im Wesentlichen ${ }^{12}$ auf die wirtschaftlichen Grundfreiheiten, aber in ihrer Wirkung auf die Mitgliedstaaten war deren Reichweite keineswegs auf Materien des Wirtschaftsrechts im engeren Sinne beschränkt und konnte deshalb auch durch die Gesetzgebung zur Vollendung des Binnenmarktes weder konsumiert noch eingegrenzt werden. Seit den Verträgen von Maastricht und Amsterdam gilt dies nun im Prinzip auch für die Rechtsprechung zu den nicht-wirtschaftlichen Freizügigkeitsrechten, Diskriminierungsverboten und Bürgerrechten. ${ }^{13}$

Mit der Direktwirkung und dem Vorrang des Europarechts werden nicht nur die Wirtschaftsfreiheiten, sondern alle aus den Verträgen abgeleiteten subjektiven Rechte „konstitutionalisiert“ und gewinnen jedenfalls im Verhältnis zum nationalen Recht die Qualität von Grundrechten. Und ebenso wie die Grundrechte der Bundesverfassung auch von den Ländern und Kommunen respektiert werden müssen, wird auch die Reichweite der europäischen Rechte nicht durch die Kompetenzen der Union und das Prinzip der „begrenzten Einzelermächtigung“ eingeschränkt. Daran können im Prinzip weder die Subsidiaritätsklausel (Art. 5 EGV) noch sogar ein expliziter Ausschluss von EU-Kompetenzen etwas ändern. So konnte etwa das Gericht Streiks in Finnland ${ }^{14}$ und Schweden ${ }^{15}$ und gesetzliche Lohnregelungen in Deutschland ${ }^{16}$ und Luxemburg ${ }^{17}$ als Verletzung der Wirtschaftsfreiheiten untersagen, obwohl doch der Amsterdam-Vertrag in Art. 137 Abs. 6 EGV eine Zuständigkeit für ,das Arbeitsentgelt, das Koalitionsrecht, das Streikrecht sowie das Aussperrungsrecht" explizit ausgeschlossen hatte. Nach der Rechtsprechung des EuGH liegt darin kein Widerspruch: Selbstverständlich behalten die Mitgliedstaaten in den genannten Politikfeldern die volle Gesetzgebungskompetenz, aber sie müssen bei deren Ausübung eben die europäischen Wirtschaftsfreiheiten und andere aus den Verträgen abgeleiteten Individualrechte

12 Von Anfang an galt dieser Schutz aber auch der Gleichstellung von Männern und Frauen im Arbeitsleben (Art. 141 EGV).

13 Wollenschläger, F.: Grundfreiheit ohne Markt. Die Herausbildung der Unionsbürgerschaft im unionsrechtlichen Freizügigkeitsregime, Tübingen, 2006.

14 EuGH, Urt. v. 11. 12. 2007, Rs. 438/05 (Viking), Slg. 2007, I-10779.

is EuGH, Urt. v. 18. 12. 2007, Rs. 341/05 (Laval), Slg. 2007, I-11767.

16 EuGH, Urt. v. 03.04. 2008, Rs. 346/06 (Rüffert), Slg. 2008, I-1989.

17 EuGH, Urt. v. 19.06. 2008, Rs. 319/06 (Luxemburg), Slg. 2008, I-4323. 
beachten. ${ }^{18}$ Kurz, über die Grundrechts-Rechtsprechung kann das Europarecht im Prinzip auf alle Zuständigkeiten der Mitgliedstaaten einwirken.

\section{Die Dynamik der negativen Integration}

Für die Wirkung der „Integration durch Recht" kommt es deshalb zunächst darauf an, wie der EuGH den Geltungsbereich der aus dem Europarecht abgeleiteten Individualrechte definiert. Die Antwort hat eine materiell-rechtliche und eine prozedurale Seite. Materiellrechtlich gibt die schon zitierte Dassonville-Formel dem Gericht die fast unbegrenzte Freiheit, in immer neuen Politikfeldern nationale Maßnahmen daraufhin zu überprüfen, ob diese die Ausübung eines europäischen Individualrechts ,direkt oder indirekt, tatsächlich oder potentiell“ behindern könnten. Zugleich gibt die Cassis-Formel dem Gericht die Freiheit, die „Zwingenden Erfordernisse des Allgemeininteresses“, mit denen der Mitgliedstaat seine Maßnahme allenfalls rechtfertigen könnte, restriktiv zu definieren und die Bedingungen festzulegen, unter denen er gegebenenfalls die Wirksamkeit und die „Verhältnismäßigkeit“ der gewählten Maßnahme beweisen müsste. Von den Mitgliedstaaten und dem Europäischen Parlament könnten die durch Richterrecht statuierten Regeln, da sie ja auf die Interpretation des Vertragsrechts gegründet werden, nur durch explizite Vertragsänderungen korrigiert werden, die jeweils einstimmig beschlossen und in allen Mitgliedstaaten ratifiziert werden müssten. Faktisch ist die EuGH-Rechtsprechung also gegen politische Korrekturen geschützt.

Ob und wie dieser potentielle Spielraum des Richterrechts, der erheblich weiter ist als der aller nationalen Verfassungsgerichte, tatsächlich genutzt wird, hängt nicht nur von der juristischen Dogmatik, sondern auch von prozeduralen Bedingungen ab, die darüber entscheiden, welche Fragen (und deshalb welche Interessen) den EuGH erreichen können. Im Vordergrund stehen dabei das Vertragsverletzungsverfahren auf Betreiben der Kommission und die Vorabentscheidung auf Vorlage der nationalen Gerichte. Im ersten Falle liegt es im politischen Ermessen der Kommission, ob sie mutmaßliche Verstöße ignorieren oder beanstanden und notfalls vor den EuGH bringen will. Im zweiten (und quantitativ weit überwiegenden) Fall liegt die Initiative formal bei den vorlegenden nationalen Gerichten,

18 So etwa in der Kohll-Entscheidung, die die Dienstleistungsfreiheit im Gesundheitswesen durchsetzte, obwohl dessen für „Organisation" nach Art. 152 Abs. 5 EGV die Verantwortung der Mitgliedstaaten ,in vollem Umfang gewahrt“ bleiben soll: EuGH, Urt. v. 28.04.1998, Rs. 158/96 (Kohll), Slg. 1998, I- 
in der Sache aber bei jenen mobilen Unternehmen und Personen, die ein starkes Interesse an der Nutzung der europäischen Wirtschaftsfreiheiten, an freiem Wettbewerb und an der Beseitigung diskriminierender Regelungen haben, und die finanziell und organisatorisch in der Lage sind, dieses Interesse auch auf dem Rechtsweg zu verfolgen. ${ }^{19}$ Keinen vergleichbaren Zugang zum EuGH haben dagegen die Interessen der nicht-mobilen Mehrheit der Bevölkerung und die Interessen, die durch die vor Gericht angegriffenen nationalen Regelungen begünstigt werden.

Diese prozedurale Asymmetrie des Zugangs hat dann wiederum eine asymmetrische Dynamik des materiellen Europarechts zur Folge: Angesichts des weiten Spielraums der Vertragsinterpretation lohnt es sich für potente Interessenten durchaus, aus ihrer Sicht unerwünschte nationale Regelungen „,auf Verdacht“ anzugreifen - und falls der EuGH dann den Schutz der Freiheits- und Mobilitätsrechte erweitert, definiert die Entscheidung einen neuen konstitutionellen status $q u o$, der im Prinzip für alle Mitgliedstaaten verbindlich ist. Zugleich werden dadurch andere Interessenten ermutigt, die mögliche Reichweite der neuen Entscheidung für die eigenen Belange zu testen, und im Erfolgsfalle die Grenze noch weiter vorzuschieben, und so fort. Zwar gibt es immer wieder auch Entscheidungen, in denen nationale Regeln trotz europarechtlicher Zweifel bestätigt werden. Sie wirken zunächst retardierend. Aber da es keinen Rechtsweg gibt, auf dem private Interessenten oder die Regierungen die restriktiven Urteile aktiv nutzen könnten, können diese auch keine gegenläufige materiellrechtliche Dynamik auslösen. Sie bleiben im Kontext der EuGH-Rechtsprechung Solitäre, aus denen sich keine Präzedenzkette entwickelt, und die deshalb aus gegebenem Anlass auch ignoriert werden können.

Aus alledem folgt eine weitere und hier entscheidende Asymmetrie des europäischen Richterrechts: Weil die Direktwirkung auf den Schutz subjektiver Rechte gegen nationale Behinderungen gegründet wurde, kann es für sich genommen nur „negative Integration ${ }^{\star 20}$ bewirken. Seine Entscheidungen untersagen die Anwendung nationaler Regelungen, die die Ausübung europarechtlich garantierter subjektiver Rechte (potentiell) behindern - sie haben also auf der nationalen Ebene notwendigerweise eine liberalisierende und deregulierende Wirkung. Das Gericht selbst wäre jedoch nicht in der Lage, das außer Kraft gesetzte nationale

19 Kelemen, R.D.: Suing for Europe. Adversarial Legalism and European Governance, in: Comparative Political Studies, 39/1 (2006), 101-127.

20 Scharpf, F.W.: Regieren in Europa. Effektiv und demokratisch?, Frankfurt/M., 1999, Kap. 2. 
Regime durch gemeinsame europäische Regeln zu ersetzen. Die ,positive Integration", die Schaffung eines europäischen Regimes zur Lösung der Probleme, die nun von den Mitgliedstaaten nicht mehr geregelt werden können, kann nur durch die europäische Gesetzgebung erreicht werden. Diese aber ist auch nach dem Übergang zu Entscheidungen mit qualifizierter Mehrheit im Ministerrat nur im breiten Konsens möglich - und sie kann deshalb nach wie vor durch gravierende Interessenkonflikte zwischen den Mitgliedstaaten oder zwischen diesen und der Kommission oder dem Europäischen Parlament blockiert werden.

Fortschritte der positiven Integration werden also auch weiterhin durch politischen Dissens behindert oder verhindert, während die negative Integration durch „unpolitisches“ Richterrecht eine gerichtete Dynamik entwickelt, bei der jede Entscheidung gegen einen Mitgliedstaat einen konstitutionellen Sperrklinkeneffekt erzeugt, während weitere Fortschritte bei der Erweiterung der europäischen Wirtschaftsfreiheiten, der Freizügigkeit und der Diskriminierungsverbote möglich und auch wahrscheinlich bleiben. Das Ergebnis ist eine progressive Beschränkung des Handlungsspielraums der demokratisch legitimierten Politik in den Mitgliedstaaten, während die - unter gegenwärtigen Bedingungen ohnehin mit schwacher demokratischen Legitimation ausgestattete ${ }^{21}$ - europäische Politik diesen Verlust an politischer Handlungsfähigkeit nicht ausgleichen kann.

\section{Die sozio-ökonomische Schlagseite der europäischen Integration}

Dieser Sachverhalt erzeugt gravierende Legitimationsprobleme im europäischen Mehrebenensystem, ${ }^{22}$ die auch nicht - anders als das Bundesverfassungsgericht im Lissabon-Urteil ${ }^{23}$ anzunehmen scheint - durch eine gegen die europäische Gesetzgebung gerichtete striktere Beachtung des Prinzips der begrenzten Einzelermächtigung gemildert werden könnten. ${ }^{24}$ Darauf will ich hier nicht eingehen. Stattdessen geht es mir um die Rückwirkungen dieser Asymmetrie von negativer

21 Follesdal, A./Hix, S.: Why there is a Democratic Deficit in the EU, in: Journal of Common Market Studies, 44/3 (2006), 533-562.

22 Scharpf, F. W.: Legitimität im europäischen Mehrebenensystem, in: Leviathan, 37/2 (2009), 244-280.

23 BVerfG, Urt. v. 30.06. 2009 (Vertrag von Lissabon), 2 BvE 2/08, 2 BvE 5/08, 2 BvR 1010/08, 2 BvR 1022/08, 2 BvR 1259/08, 2 BvR 182/09.

24 Immerhin will aber das Bundesverfassungsgericht seine eigene „Ultra-vires und Identitätskontrolle“ auch auf die Auslegung europäischer Zuständigkeiten erstrecken (ebd., Rz. 242). Ob damit freilich auch die Interpretation der Reichweite europäischer Freiheitsrechte und Diskriminierungsverbote gemeint ist, bleibt abzuwarten. 
und positiver Integration auf die sozioökonomischen Strukturen der Mitgliedstaaten.

Freilich ist die Schwäche der positiven Integration nicht auf allen Politikfeldern gleichermaßen spürbar: Im Verbraucherschutz, im Arbeitsschutz und auch im Umweltschutz gibt es in der Tat viele Beispiele für außerordentlich stringente und auch wirksame europäische Verordnungen und Richtlinien - man denke nur an das jüngste Verkaufsverbot für Glühlampen. ${ }^{25}$ Die Union ist durchaus handlungsfähig, wo die mitgliedstaatlichen Interessen gleichgerichtet sind oder wo Entscheidungen in der „Komitologie“ entpolitisiert werden können. Aber wenn die Mitgliedstaaten bei politisch sichtbaren und wichtigen Entscheidungen unterschiedliche oder sogar gegensätzliche Interessen verteidigen, sind wirksame europäische Problemlösungen nicht zu erwarten und es bleibt dann bei der richterrechtlichen Deregulierung nationaler Lösungen. Diese aber trifft die Mitgliedstaaten und deren überkommene Wirtschafts- und Gesellschaftsstrukturen in ganz unterschiedlichem Maße.

Um dies zu erkennen bedarf es eines Blicks auf die Ergebnisse der vergleichenden sozio-ökonomischen Forschung. Diese hat in den letzten beiden Jahrzehnten die westlichen Industriestaaten in zwei Dimensionen - der polito-ökonomischen und der sozio-politischen - klassifiziert. In der ersten Dimension unterscheidet die varieties-of-capitalism-Forschung zwei unterschiedliche Typen der marktwirtschaftlichen Strukturen, die „,coordinated market economies “ und die ,liberal market economies " ${ }^{26}$ In den koordinierten Marktwirtschaften wird der $\mathrm{Zu}$ gang der Unternehmen zu den wichtigen Produktionsfaktoren - Kapital, Arbeit, Qualifikation, Technologie - durch kooperative Dauerbeziehungen und staatliche Regeln und Leistungen stabilisiert, während in den liberalen Marktwirtschaften die selben Funktionen im Wesentlichen durch den Markt erfüllt werden. In der ökonomischen Theorie des freien Handels werden diese grundlegenden institutionellen Unterschiede als „komparative Vorteile“ interpretiert, die die wirtschaftliche Leistungsfähigkeit des betreffenden Standorts für jeweils unterschiedliche

25 Verordnung (EG) Nr. 244/2009 der Kommission vom 18.03.2009 zur Durchführung der Richtlinie 2005/32/EG des Europäischen Parlaments und des Rates im Hinblick auf die Festlegung von Anforderungen an die umweltgerechte Gestaltung von Haushaltslampen mit ungebündeltem Licht, ABIEU Nr. L $76 / 3$ v. 24.03. 2009.

26 Hall, P./Soskice, D.: Varieties of Capitalism: The Institutional Foundations of Comparative Advantage, Oxford, 2001; Hancké, B./Rhodes, M./Thatcher, M. (Hg.): Beyond Varieties of Capitalism: Conflict, Contradictions, and Complementarities in the European Economy, Oxford, 2007; Höpner, M.: „Spielarten des Kapitalismus" als Schule der vergleichenden Staatstätigkeitsforschung, in: Zeitschrift für vergleichende Politikwissenschaft, 3/2 (2009), 303-327. 
Wirtschaftszweige entweder begünstigen oder behindern. Dem entspricht der empirische Befund, dass die Stärken der koordinierten und der liberalen Marktwirtschaften auf unterschiedlichen Märkten liegen, und dass bei gegebener Kongruenz zwischen Branchenstruktur und institutioneller Struktur Länder aus der einen wie aus der anderen Gruppe im internationalen Wettbewerb höchst erfolgreich sein können.

Die zweite Klassifikation liefert die vergleichende Sozialstaatsforschung. ${ }^{27}$ Sie unterscheidet idealtypisch zwischen der „liberalen“, der „,christdemokratischen“ und der ,sozialdemokratischen“ Ausprägung des „welfare capitalism “, ${ }^{28}$ die jeweils in den angelsächsischen, den kontinentaleuropäischen und den skandinavischen Ländern annähernd realisiert werden. Ihre Gemeinsamkeit besteht darin, dass überall eine steuerfinanzierte Mindestversorgung bei Armut und im Gesundheits- und Bildungswesen gewährleistet wird. Aber während die liberalen Staaten sich im Wesentlichen darauf beschränken und weitergehende Sicherungs- und Betreuungsleistungen der privaten Vorsorge und dem Markt überlassen, sorgen die christdemokratischen und sozialdemokratischen Sozialstaaten auch für die Absicherung des durch die Erwerbsarbeit erreichten Einkommens bei Arbeitslosigkeit und im Alter. Darüber hinaus sorgen die sozialdemokratischen Sozialstaaten auch für ein umfassendes Angebot sozialer Dienstleistungen für Kinder, Kranke, Behinderte und Pflegebedürftige, die die Familien von Betreuungs- und Pflegeleistungen entlasten, und die in den anderen beiden Modellen entweder von den Frauen in der Familie oder vom Markt erbracht werden. Dementsprechend ist die Abgabenlast hier am höchsten und in den liberalen Sozialstaaten am geringsten, und bei der sozialen Ungleichheit gilt die umgekehrte Rangfolge.

Beide Klassifikationen verwenden freilich vereinfachende Idealtypen. Nicht jedes Land, das dem einen oder anderen cluster zugeordnet wird, weist alle diesem zugeschriebenen Merkmale auf. Und da die beschriebenen Strukturen ihre volle Ausprägung in den Nachkriegsjahrzehnten erhielten, wird die gegenwärtige Trennschärfe der Kategorien auch durch unterschiedliche Reaktionen der einzelnen Länder auf neue Herausforderungen vermindert. ${ }^{29}$ Trotzdem will ich hier die Vereinfachung noch weiter treiben und die polit-ökonomische und die sozio-

27 Alber, J./Gilbert, N. (Hg): United in Diversity? Comparing Social Models in Europe and America, Oxford, 2009.

28 Esping-Andersen, G.: The Three Worlds of Welfare Capitalism, Princeton, 1990.

29 Scharpf, F.W./Schmidt, V.A. (Hg.): Welfare and Work in the Open Economy, 2 Bde., Oxford, 2000. 
politische Klassifikationen zu einer einzigen zusammenfassen, die unter den Mitgliedstaaten der EU nur noch zwischen ,sozialen Marktwirtschaften“ und „liberalen Marktwirtschaften“" unterscheidet. Zur zweiten Gruppe zähle ich dann die angelsächsischen und osteuropäischen „liberalen Marktwirtschaften“ deren sozialstaatliche Leistungen ebenfalls dem ,liberalen Modell“ entsprechen. Die Gruppe der ,sozialen Marktwirtschaften“ umfasst dann die kontinentalen und skandinavischen Länder mit einer „koordinierten“ Wirtschaftsstruktur, deren sozialstaatliche Leistungsstrukturen entweder dem „christdemokratischen“ oder dem ,sozialdemokratischen“" Modell entsprechen.

Auf dieser konzeptionellen Grundlage lässt sich nun ein zweidimensionaler Politikraum definieren, in dem die vertikale Dimension die Differenz zwischen nationaler politischer Autonomie und der vollständigen Europäisierung der Kompetenzen bezeichnet, während die horizontale Dimension die Differenz zwischen „sozialen“ und „liberalen" sozioökonomischen Strukturen beschreibt. Solange die europäische Integration die politische Autonomie der Mitgliedstaaten noch nicht spürbar eingeschränkt hatte, waren die Mitgliedstaaten je nach ihrer sozioökonomischen Struktur in der unteren Hälfte des Feldes zu lokalisieren - die

Abbildung 1: Sozioökonomische Strukturen im Europäisierungsprozess

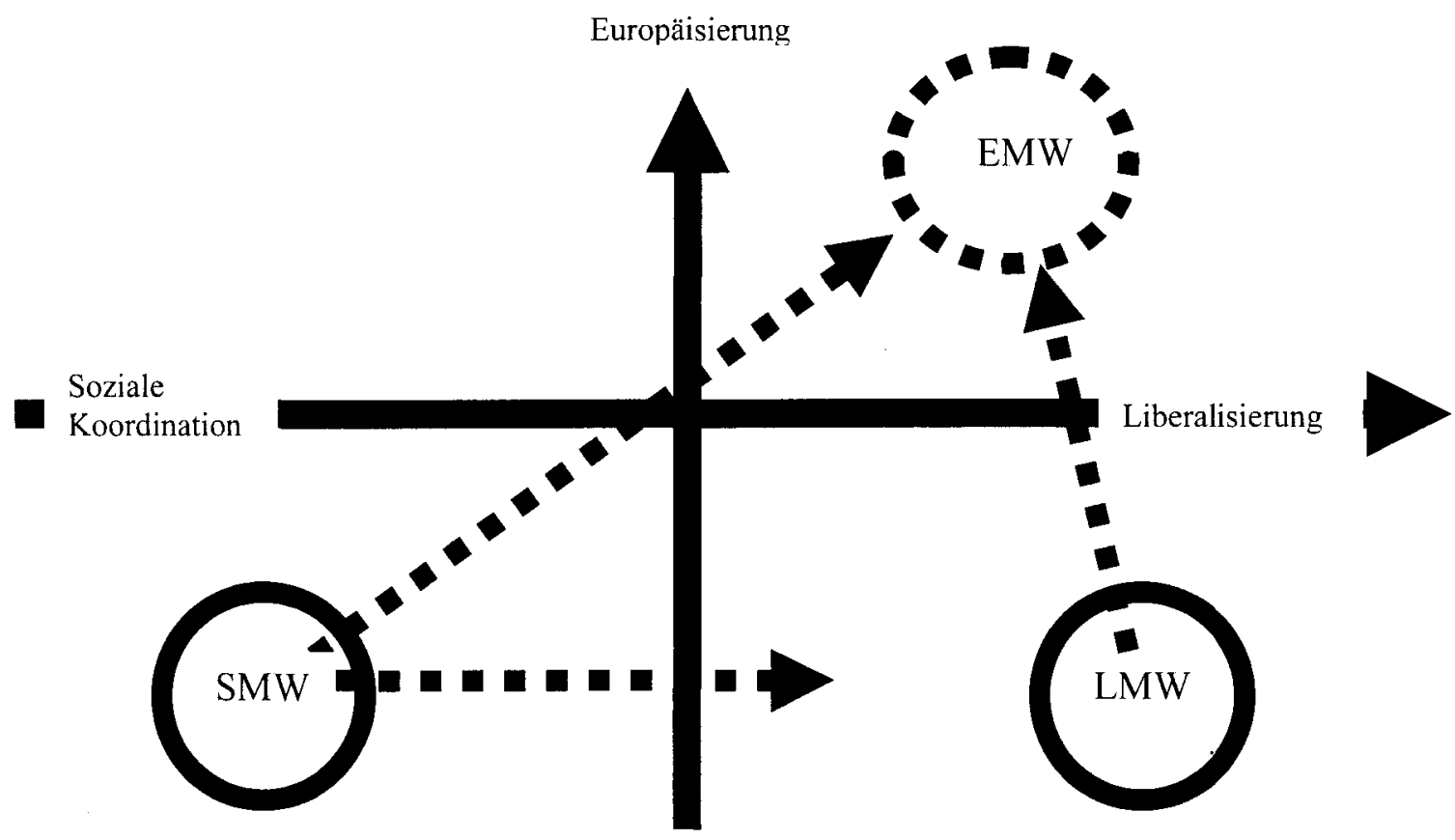

Nationale Autonomie

Quelle: Eigene Darstellung 
„Sozialen Marktwirtschaften“ (SMW) im linken und die „liberalen Marktwirtschaften" (LMW) im rechten unteren Quadranten (vgl. Abbildung 1).

Aus dem zuvor Gesagten folgt nun, dass die marktnahen Wirtschafts- und Sozialstrukturen der liberalen Marktwirtschaften, und damit auch deren bisherige rechtliche Ordnung von der liberalisierenden und deregulierenden Rechtsprechung des EuGH nur wenig betroffen wird. Im Gegenteil, diese Länder (zu denen neben Großbritannien, Irland und oft den Niederlanden auch viele der neuen Mitgliedstaaten in Mittel- und Osteuropa gehören) haben gute Gründe, die Beseitigung von nicht-tarifären Handelshindernissen und die Zulassung des Wettbewerbs in den zuvor geschützten Aufgabenfeldern der übrigen Länder zu begrüBen. Sie profitieren insgesamt von der negativen Integration und werden diese auch in der europäischen Gesetzgebung verteidigen und weiter vorantreiben.

Ganz anders die Situation der sozialen Marktwirtschaften. Die Institutionen und Regeln, auf denen die komparativen Vorteile ihrer Wirtschaftsstruktur beruhen, werden durch den Deregulierungseffekt der negativen Integration zum Teil außer Kraft gesetzt und durch reine Marktmechanismen verdrängt. Zugleich hat das europäische Wettbewerbsrecht frühere Instrumente der staatlichen Strukturpolitik beseitigt oder - so etwa die strukturpolitische Funktion öffentlicher Banken geschwächt. Zugleich eröffnet die Rechtsprechung zur Niederlassungsfreiheit den Unternehmen die Möglichkeit, das Gesellschaftsrecht ihres Sitzlandes durch Ausgründung in einem anderen Mitgliedstaat zu vermeiden ${ }^{30}$ und wenn das Urteil zum Volkswagengesetz schon die Festlegung einer Sperrminorität in der Hauptversammlung als unzulässige Behinderung des freien Kapitalverkehrs gewertet hat, ${ }^{31}$ dann steht auch die Mitbestimmung der Arbeitnehmer im Aufsichtsrat in Frage, sobald der der EuGH dazu kommt, diese an den europäischen Freiheitsrechten zu messen. Kurz: Im Gegensatz zur ökonomischen Theorie schützt die europäische Rechtsprechung nicht den internationalen Wettbewerb zwischen Ländern mit unterschiedlichen komparativen Vorteilen, sondern erzwingt die Konvergenz aller Mitgliedstaaten auf das Modell einer liberalen Marktwirtschaft. ${ }^{32}$

30 EuGH, Urt. v. 09.03.1999, Rs. 212/97 (Centros), Slg. 1999, I-1459; Roth, G. H./Demetz, M./Donath G.: Gesellschaftsrecht: Briefkastengründungen und Golden Shares, in: Roth, G. H./Hiltpold, P. (Hg.): Der EuGH und die Souveränität der Mitgliedstaaten, Wien, 2008, 427-480.

31 EuGH, Urt. v. 23. 10. 2007, Rs. 112/05 (Deutschland), Slg. 2007, I-8995.

32 Höpner, M./Schäfer, A.: A New Phase of Integration: Organized Capitalism in Post-Ricardian Europe, MPIfG Discussion Paper 07/04, Köln, 2007. 
Ähnlich steht es um die Auswirkungen der negativen Integration auf die Sozialpolitik der Mitgliedstaaten. Wiederum werden die liberalen Sozialstaaten von den Liberalisierungseffekten der europäischen Rechtsprechung nur wenig betroffen. Die für sie charakteristischen Leistungen einer privaten Gesundheits- und Altersvorsorge und privater Pflege und Betreuungsdienste brauchen nicht mehr liberalisiert zu werden. Allenfalls kann es bei den steuerfinanzierten Leistungen der Sozialhilfe (und im britischen Fall bei den Leistungen des staatlichen Gesundheitsdienstes) zu Konflikten mit der Rechtsprechung zum Diskriminierungsverbot und zur Dienstleistungsfreiheit kommen. ${ }^{33}$

Die christ- und sozialdemokratischen Sozialstaaten dagegen nutzen bisher eine Vielfalt von Regelungs-, Finanzierungs- und Erbringungsformen. Soziale Dienstleistungen können öffentlich erbracht und aus dem allgemeinen Steueraufkommen oder über Gebühren finanziert werden, oder sie können privat erbracht und öffentlich subventioniert, über eine öffentliche Zwangsversicherung oder eine subventionierte private Versicherung finanziert werden, und Ähnliches gilt für die steuerfinanzierten, auf Zwangsversicherung gestützten oder privaten und staatlich subventionierten Formen der Altersvorsorge und andere Transfers. Wo sie öffentliche und private Elemente verbinden, steht ihre Vereinbarkeit mit dem europäischen Wettbewerbs- und Subventionsrecht grundsätzlich in Frage. Überdies erfordern die christdemokratischen und erst recht die sozialdemokratischen Sozialstaaten einen hohen fiskalischen Aufwand. Sie sind deshalb auch besonders anfällig für den Wettbewerbsdruck auf die Kapitalbesteuerung, der sie wenn sie das Leistungsniveau erhalten wollen - zur politisch prekären und beschäftigungsschädlichen Verlagerung der Lasten auf weniger mobile Steuerquellen und lohnbasierte Sozialabgaben zwingt.

Vor allem aber erfordert das höhere Leistungsniveau differenzierende Regeln zur Abgrenzung der Leistungsarten, der Leistungsempfänger, der Leistungsvoraussetzungen und der zugelassenen Leistungserbringer - und diese Regeln können im Prinzip immer mit den europäischen Wirtschaftsfreiheiten, dem Wettbewerbsrecht, der Freizügigkeit und dem Diskriminierungsverbot in Konflikt geraten. Tatsächlich hat die neuere Rechtsprechung privaten Leistungsanbietern den Zugang zu kollektiv finanzierten und organisatorisch geschlossenen nationalen Systemen eröffnet, sie sichert die Kostenerstattung, wenn die Klienten nationaler Systeme auf Leistungen im Ausland ausweichen, und sie beschränkt in zuneh-

33 Martinsen, D.S.: Conflict and Conflict Management in the Cross-Border Provision of Health Care Services, in: West European Politics, 32/4 (2009), 792-809. 
mendem Maße die Möglichkeit, zugewanderte EU-Ausländer vom Zugang zu steuerfinanzierten nationalen Leistungen auszuschließen. ${ }^{34}$ Eine Rechtsprechung, die auf diese Weise den Schutz subjektiver Individualrechte einseitig vorantreibt, kann zugleich das Gleichgewicht zwischen Beiträgen und Leistungen kollektiver Sozialsysteme untergraben.

Ein eklatantes Beispiel dafür bietet die Entscheidung, die deutschen Medizinstudenten, die vor dem numerus clausus und den heimischen Studiengebühren an österreichische Fakultäten flüchten, dort den freien Zugang sicherte ${ }^{35}-$ mit der Folge, dass danach mehr als $60 \%$ der Studienbewerber aus Deutschland kamen. Eine solche Anwendung des Diskriminierungsverbots ignoriert die von der nationalen Politik gewollten und demokratisch legitimierten Unterschiede in der mehr oder weniger solidarischen Gestaltung gesellschaftlicher Leistungssysteme. Hätte Österreich den Zugang zu seinen Universitäten durch hohe Studiengebühren rationiert, hätte es keine europarechtliche Beanstandung gegeben, und das Gericht selbst wies darauf hin, dass die behauptete Überfüllung der Medizinfakultäten ja durch eine einheitliche Zulassungsprüfung hätte abgewendet werden können. Dass die österreichische Politik aber aus gesellschaftspolitischen Gründen die Studierneigung der einheimischen Abiturienten fördern und aus gesundheitspolitischen Gründen die Zahl der einheimischen Ärzte erhöhen wollte, erschien unter dem Kriterium des Diskriminierungsverbots völlig irrelevant. Man kann dieses Urteil, ebenso wie die Entscheidungen zur Inanspruchnahme nationaler Sozialleistungen durch Gebietsfremde als ein der „Unionsbürgerschaft“ dienendes „Freizügigkeitsregime“ feiern. ${ }^{36}$ Aber solange es dabei nicht um gemeinsam beschlossene und gemeinsam finanzierte europäische Leistungen geht, zerreißt diese Rechtsprechung den normativen Zusammenhang von Bürgerrechten und Bürgerpflichten und untergräbt die Verantwortung der nationalen Politik für die Definition solidarischer Leistungen und die dafür von den eigenen Bürgern einzufordernden Beiträge. Im Ergebnis müssen also Sozialstaaten mit einem hohen Leistungsniveau sich in dem europäischen Freizügigkeitsregime entweder ausbeuten lassen, oder sie müssen ihre Leistungen auch für die eigenen Bürger so weit senken, dass ein Anreiz für ,,welfare migration“ vermieden wird. ${ }^{37}$ ance: Implementing the Market Imperatives of Europe, in: Public Administration, 86/1 (2008), 169-184.

35 EuGH, Urt. v. 07.07. 2005, Rs. 147/03 (Österreich), Slg. 2005, I-5969.

36 Wollenschläger, $F$., a. a. O.

37 Menéndez, A.J.: European Citizenship after Martinez Sala and Baumbast. Has European Law Become More Human and Less Social?, RECON Online Working Paper 2009/05, Oslo. 
Ebenso wie bei der Wirtschaftsstruktur erzwingt also auch bei der Sozialstruktur die durch europäisches Richterrecht durchgesetzte negative Integration strukturelle Änderungen der nationalen Systeme. Diese untergraben in asymmetrischer Weise die Institutionen und die politische Praxis der "sozialen Marktwirtschaft", während die Strukturen der „liberalen Marktwirtschaft" von der negativen Integration kaum betroffen werden. Im Ergebnis kommt es deshalb auch hier zu einer tendenziellen Angleichung der höchst unterschiedlichen nationalen Strukturen an das Modell einer liberalen Marktwirtschaft. In Abbildung 1 verschiebt sich deshalb der Ort der SMW-Länder vom unteren linken zum unteren rechten Quadranten.

Offenbar haben die christ- und sozialdemokratischen Regierungen in der EU diesen Effekt inzwischen erkannt, und sie haben es im Rahmen der Verhandlungen über den Lissabon-Vertrag sogar geschafft, zu den „Zielen der Union“ auch „eine in hohem Maße wettbewerbsfähige soziale Marktwirtschaft, die auf Vollbeschäftigung und sozialen Fortschritt abzielt" hinzuzufügen (Art. 3, Abs. 3, S. 2 EUV). Aber wie alle auf positive Integration gerichteten Ziele könnte auch dieses nicht durch das Richterrecht, sondern nur durch die europäische Gesetzgebung erreicht werden. Auf diese käme es an, wenn die in den Mitgliedstaaten erodierenden Strukturen einer sozialen Marktwirtschaft auf der europäischen Ebene neu geschaffen werden sollten. Dafür stehen die Chancen jedoch ebenso ungünstig, wie dies zuvor für das ,europäische Sozialmodell“ oder für die von Jacques Delors geforderte „soziale Dimension“ des Binnenmarktprogramms der Fall war. Und der Grund liegt heute wie damals im hohen Konsensbedarf der europäischen Politik. Dieser begünstigt den status quo überall dort, wo Entscheidungen nicht unterhalb der öffentlichen Aufmerksamkeit in unpolitischen Gremien ausgehandelt werden können, und wo die nationalen Interessen, Weltsichten und normativen Wertungen stark divergieren. Aber der hier relevante status quo ist nicht die bestehende Praxis der einzelnen Mitgliedstaaten, sondern die durch das europäische Richterrecht veränderte Ausgangslage.

Wo das Richterrecht die Regeln der sozialen Marktwirtschaften außer Kraft setzt, entspricht dies zumeist der bisherigen Praxis und den politischen Präferenzen der liberalen Marktwirtschaften, die deshalb auch keinen Grund haben, dieses Ergebnis zugunsten einer sozialen Marktwirtschaft auf europäischer Ebene korrigieren zu wollen. Einigen können sich beide Lager allenfalls auf europäische Verordnungen und Richtlinien, die die punktuell liberalisierenden EuGH- 
Entscheidungen systematisieren und generalisieren, um so die andernfalls bestehende Rechtsunsicherheit für Verwaltungen und Unternehmen zu vermeiden. ${ }^{38}$ Europäische Gesetzgebung, die die positive Integration vorantreibt ist dagegen vor allem in Bereichen zu erwarten, in denen das Gericht nationale Regeln als ,zwingende Erfordernisse des öffentlichen Interesses“ anerkannt hat, und in denen deshalb die europäische Harmonisierung notwendig bleibt, wenn die Fragmentierung nationaler Märkte überwunden werden soll. Dies gilt insbesondere für Regeln des Verbraucherschutzes, Umweltschutzes und Arbeitsschutzes, aber gerade nicht für die Unternehmensverfassung, die Kapitalbesteuerung, die Arbeitsbeziehungen, die sozialen Dienste und die Infrastrukturfunktionen, die für die Funktionsweise der sozialen Marktwirtschaften von grundlegender Bedeutung waren und sind. In allen diesen Feldern sind die bisherigen Versuche einer Europäisierung gescheitert und nichts spricht dafür, dass dies sich unter dem Lissabon-Vertrag ändern würde.

Es bleibt also bei dem in Abbildung 1 dargestellten Befund: Die negative Integration durch das politisch nicht korrigierbare europäische Richterrecht lässt die „liberalen Marktwirtschaften“ weitgehend unberührt, aber sie untergräbt die Institutionen und die politische Praxis der sozialen Marktwirtschaft und sie bewirkt deren Annäherung an die Strukturen der liberalen Marktwirtschaft. Der Lissabon-Vertrag formuliert nun das Ziel, die Strukturen einer sozialen Marktwirtschaft auf der europäischen Ebene zu institutionalisieren. Dies könnte nicht durch Richterrecht, sondern nur durch europäische Gesetzgebung - und deshalb nur im breiten politischen Konsens - erreicht werden. Da aber der Gegensatz zwischen den Zielen und den Institutionen einer liberalen und einer sozialen Marktwirtschaft in allen Mitgliedstaaten von hoher ideologischer und politischpragmatischer Bedeutung ist, kann die europäische Politik das postulierte Ziel nicht im Konsens erreichen. Wer also die soziale Marktwirtschaft in den Mitgliedstaaten verteidigen will, sollte seine Hoffnung nicht auf die europäische Gesetzgebung richten, sondern nach Lösungen suchen, die die destruktive Wirkung des europäischen Richterrechts begrenzen können. 